J. Clin. Chem. Clin. Biochem.

Vol 19, 1981, pp. 203-208

\title{
Quantitative Proteinbestimmung im Harn mit dem Proteinbindungsfarbstoff Coomassie Brilliant Blau G250
}

\author{
Von L. Thomas, M. Winckelmann, H. C. Michaelis und D. Walb \\ Zentrallaboratorium und Fachbèreich Nephrologie der Deutschen Klinik für Diagnostik Wiesbaden
}

(Eingegangen am 2. Juli 1979/21. November 1980)

\begin{abstract}
Zusammenfassung: Die quantitative Bestimmung von Harnprotein unter Anwendung des Proteinbindungsfarbstoffes Coomassie Brilliant Blau G250 wird beschrieben. Vergleichend erfolgte die Durchführung der Biuret-Reaktion. Zur Bestimmung werden 0,10 ml Harnprobe mit 5,0 ml Coomassie Brilliant Blau G250-Farbreagenz versetzt und die Absorptionszunahme des Ansatzes gegenüber dem Farbreagenz gemessen. Aufgrund besserer Präzision, höherer Empfindlichkeit und einfacherer Durchführung ist die Coomassie Brilliant Blau G250-Methode bei der Proteinbestimmung im Harn der Biuret-Reaktion überlegen. In einem Kollektiv von 49 klinisch gesunden Probanden liegt die obere Normalbereichsgrenze der Proteinausscheidung bei $120 \mathrm{mg} / 24 \mathrm{~h}$.
\end{abstract}

Zur Beurteilung der Wertigkeit der Coomassie Brilliant Blau G250-Methode für die Proteinausscheidung im Harn von Kranken wurde die Proteinkonzentration von 134 Patienten mit Organleiden, Stoffwechsel- und Systemerkrankungen vergleichend zur Biuret-Reaktion beurteilt. Der Vergleich beider Methoden ergibt eine Regressionsgerade von $y=0,827 x+8,713 \mathrm{mg} / \mathrm{l}$ mit einem Korrelationskoeffizienten von $\mathrm{r}=0,966$. Der Typ der Proteinurie (gemischt, glomerulär, tubulär) ist nicht maßgebend für die Höhe des mit der Coomassie Brilliant Blau G250-Methode gemessenen Proteinwertes, solange die selektive Ausscheidung eines Einzelproteins (z. B. Bence-Jones-Protein) nicht stark überwiegt. Diabetiker zeigen gehäuft niedrigere Werte mit der Coomassie Brilliant Blau G250-Methode, Dialysepatienten gehäuft erhöhte. Trotz dieser Einschränkungen könnte die Coomassie Brilliant Blau G250-Methode das Verfahren der Wahl für die routinemäßige Geșamteiweißbestimmung im Urin in der klinisch-chemischen Diagnostik werden.

\section{Quantitative determination of total urinary protein utilizing the principle of Coomassie Brilliant Blue G250 binding to protein}

Summary: Coomassie Brilliant Blue G250 was used for the determination of total urinary protein and compared with the biuret-procedure. In the Coomassie Brilliant Blue $\mathrm{G} 250$-assay $0.10 \mathrm{ml}$ urine are added to $5.0 \mathrm{ml}$ Coomassie Brilliant Blue G250 reagent and the sample is read against the reagent blank. The Coomassie Brilliant Blue G250 method seems to be superior to the biuret-procedure because of better reproducibility, higher sensitivity and simpler handling. The upper limit of urinary protein excretion in 49 healthy subjects was $120 \mathrm{mg} / 24 \mathrm{~h}$.

To evaluate the clinical significance of the Coomassie Brilliant Blue G250 method the urinary protein concentration of 134 patients with metabolic, systemic and organ diseases was compared with the biuret-procedure. The regression line was $y=0.827 x+8.713 \mathrm{mg} / 1$ with a correlation coefficient of $\mathrm{r}=0.966$. The type of proteinuria (mixed, glomerular, tubular) had no influence on the protein value measured with the Coomassie Brilliant Blue G250 method. However in selective proteinuria, like Bence-Jones protein excretion, there is no correlation between the two methods, because the concentration of Bence-Jones protein is underestimated. Lower protein values were also frequently obtained for patients with diabetes mellitus. Some specimens from patients with chronic renal failure treated by haemodialysis showed elevated values. In spite of these limitations the Coomassie Brilliant Blue G250 method might become the method of choice for the determination of total urinary protein.

\section{Einführung}

Die quantitative Bestimmung der Proteinausscheidung im Harn ist ein wichtiges Kriterium zur Diagnostik von
Nierenerkrankungen. Sie wird den Teststreifenverfahren zum Nachweis von Protein (Nachweisempfindlichkeit $150 \mathrm{mg} / \mathrm{l}$ bezogen auf Albumin) nachgeschaltet, wenn 
diese eine Proteinurie anzeigen oder das Ergebnis nicht mit dem klinischen Befund in Einklang zu bringen ist. Die am häufigsten angewendeten Verfahren zur quantitativen Proteinbestimmung im Harn sind die BiuretReaktion $(1,2,3,4)$, turbidimetrische Techniken (5, $6)$, die Folin-Lowry-Reaktion $(7,8)$ und die SephadexBiuret-Methode (9). Nachteile dieser Methoden sind entweder die schwierige Durchfuhrbarkeit (SephadexBiuret-Methode) oder eine mangelnde Spezifität (FolinLowry-Reaktion). Auch werden nicht alle Proteine erfaßt (Turbidimetrie) oder die Empfindlichkeit ist zu gering (Biuret-Reaktion). Die oberen Normalbereichsgrenzen der 24 h-Proteinausscheidung differieren bis um den Faktor $5(10,3)$.

Im Jahre 1976 hat M. Bradford (11) die Messung kleiner Proteinmengen mit dem Textilfarbstoff Coomassie Brilliant Blau G250 beschrieben. In saurer Lösung ist dieser Farbstoff rot gefärbt (Absorptionsmaximum bei $546 \mathrm{~nm}$ ). Mit Proteinen reagiert Coomassie Brilliant Blau G250 schnell unter Ausbildung eines ProteinFarbstoff-Komplexes, gewinnt dabei seine blaue Farbe zurück und hat ein Absorptionsmaximum bei $595 \mathrm{~nm}$.

Untersuchungen mehrerer Arbeitsgruppen haben gezeigt, daß die Coomassie Brilliant Blau G250-Methode für die Liquorproteinbestimmung ein einfaches, schnelles und sensitives Verfahren ist $(12,13)$. Die Meinungen über die Anwendbarkeit zur Harnproteinbestimmung sind geteilt $(12,14)$. Wir haben deshalb die Eignung der Coomassie Brilliant Blau G250-Methode zur Proteinbestimmung im Harn klinisch gesunder Probanden und von Patienten untersucht. Als Vergleichsmethode diente die BiuretReaktion.

\section{Methoden und Material}

Die angewendeten Methoden der Proteinbestimmung von Albuminlösungen und in Harnen sowie die Ankonzentrierung und elektrophoretische Trennung der Harnproteine werden nachfolgend beschrieben.

\section{Biuret-Reaktion}

Die Durchführung erfolgte mit dem Merckotest ${ }^{\circledR}$ GesamtEiweiß (E. Merck, Darmstadt) Art.-Nr. 3327. 10,0 ml Probe wurden mit $2,0 \mathrm{ml}$ Perchlorsäure. $4,5 \mathrm{~mol} / 1$ versetzt, $10 \mathrm{~min}$ im Eisbad stehen gelassen und nachfolgend $10 \mathrm{~min}$ bei $3000 \mathrm{~g}$ zentrifugiert. Nach Entnahme des Überstandes wurde das Präzipitat mit 5,0 ml Biuret-Reagenz versetzt und nach $30 \mathrm{~min}$ die Farbkonzentration bei $546 \mathrm{~nm}$ gegen den Analysenblindwert gemessen. Dieser wurde abweichend von der Behandlung des Probenansatzes mit Vergleichsreagenz angesetzt. Die Ermittlung der Proteinkonzentration erfolgte durch Erstellung einer Standardkurve mit Rinderserumalbumin.

\section{Coomassie-Brilliant-Blau G250-Methode}

$0,10 \mathrm{ml}$ Probe wurden mit $5,0 \mathrm{ml}$ Farbreagenz versetzt, durch leichtes Schwenken gemischt und im Zeitraum von 5-30 min die Farbkonzentration bei $595 \mathrm{~nm}$ gegen $5,0 \mathrm{ml}$ Farbreagenz, dem $0,10 \mathrm{ml}$ bidest. Wasser zugesetzt waren, gemessen. Von Patientenharnen, die mit den Teststreifenverfahren eine Eiweiß- reaktionen von ++ oder stärker zeigten, wurden nur $0,010 \mathrm{ml}$ zur Analyse eingesetzt. Wegen Adsorption des Farbstoffes an die Küvettenwände wurden Durchlaufküvetten nicht verwendet. Die Ermittlung der Proteinkonzentration erfolgte durch Erstellung einer Standardkurve mit Rinderserumalbumin. Die Standardkurve wurde immer neu erstellt, wenn die Herstellungscharge von Coomassie-Brilliant-Blau G250 und von Rinderserumalbumin sich änderte.

Zusammensetzung des Farbreagenzes: Coomassie-Brilliant Blau G250 0,10 g/l, o-Phosphorsäure 0,867 mol/1, Ethanol absolut $0,10 \mathrm{~mol} / \mathrm{l}$. Etwa noch vorhandene Farbstoffpartikel wurden durch Filtrieren entfernt. Haltbarkeit bei Raumtemperatur in dunkler Flasche mindestens 2 Monate. Zur Sicherung, daß immer im Absorptionsmaximum des Farbstoff-ProteinKomplexes gemessen wurde, erfolgte mit jedem Chargenwechsel von Coomassie Brilliant Blau G250 die Bestimmung des Absorptionsmaximums. Dażu wurden $5,0 \mathrm{ml}$ Farbreagenz mit $0,10 \mathrm{ml}$ Albuminlösung ( $400 \mathrm{mg} / \mathrm{l})$ versetzt und bei Wellenlängen von 580-620 nm die Absorption gemessen.

\section{Ankonzentrierung der Harneiweiß̧e}

Die Konzentrierung der Hameiweiße zum elektrophoretischen Nachweis des Hamproteinmusters erfolgte mit dem HollowFiber-Konzentrator $\mathrm{CH} 3$ von Amicön, Witten/Ruhr. Verwendet wurde die Diaflo-Hollow-Fiber-Patrone HP 10 mit einer nominellen Trenngrenze für glomeruläre Proteine von 10000 Dalton.

\section{Elektrophoretische Untersuchungen}

Zur Auffindung selektiver Bence-Jones-Proteinurien wurden Harne auf eine Proteinkonzentration von etwa $20 \mathrm{~g} / 1$ ankonzentriert und die Urineiweiß-Elektrophorese auf Celluloseacetatfolie durchgefüht (15). Der Nachweis von Bence-JonesProtein erfolgte immun-elektrophoretisch (15). Die molekulargewichtsbezogene Auftrennung und Klassifizierung der Proteeinurien in glomeruläre, tubuläre und gemischte Formen wurde mit der SDS-Polyacrylamidgel-Elektrophorese durchgefuihrt (16). Im Mittel wurden $50 \mu \mathrm{g}$ Hainprotein aufgetrennt.

\section{Reagenzien}

Rinderserumalbumin, Behringwerke, Marburg, Art.-Nr. $\phi$ RHA 04 sowie Boehringer Mannheim, Art.-Nr. 237604. Die Stammlösung von Rinderserumalbumin und die Verdünnungen wurden in bidest. Wasser hergestellt. Coomassie Brilliant Blau G250 wurde von Serva Heidelberg bezogen, Art.-Nr. 35050.

Untersuchungsmaterial

24 h-Sammelharne sowie morgendlicher Spontanharn.

\section{Ergebnisse}

Vor Anwendung der Coomassie Brilliant Blau G250Methode und der Biuret-Reaktion zur Bestimmung der Proteinkonzentration in Hamen wurden Empfindlichkeit, Linearität und Präzision beider Analysenverfahren für wäßrige Rinderserumalbuminlösungen untersucht.

\section{Analytische Eigenschaften von Coomassie} Brilliant Blau G250-Methode und Biuret-Reaktion fürr reine Proteinlösungen

\section{Linearität}

Die Biuret-Reaktion zeigt bei dem gewählten Probeneinsatz von $10.0 \mathrm{ml}$ keine Abweichung von der Linearität 
bis zu einer Albuminkonzentration von $1,2 \mathrm{~g} / \mathrm{l}$ (Abb. 1). Die Coomassie Brilliant Blau G250-Methode hat eine lineare Beziehung zwischen Proteinmenge und Absorption bis zu einer Albuminkonzentration von $0.40 \mathrm{~g} / \mathrm{l}$. Es tritt dann eine zunehmende Unlinearität auf (Abb. 2).

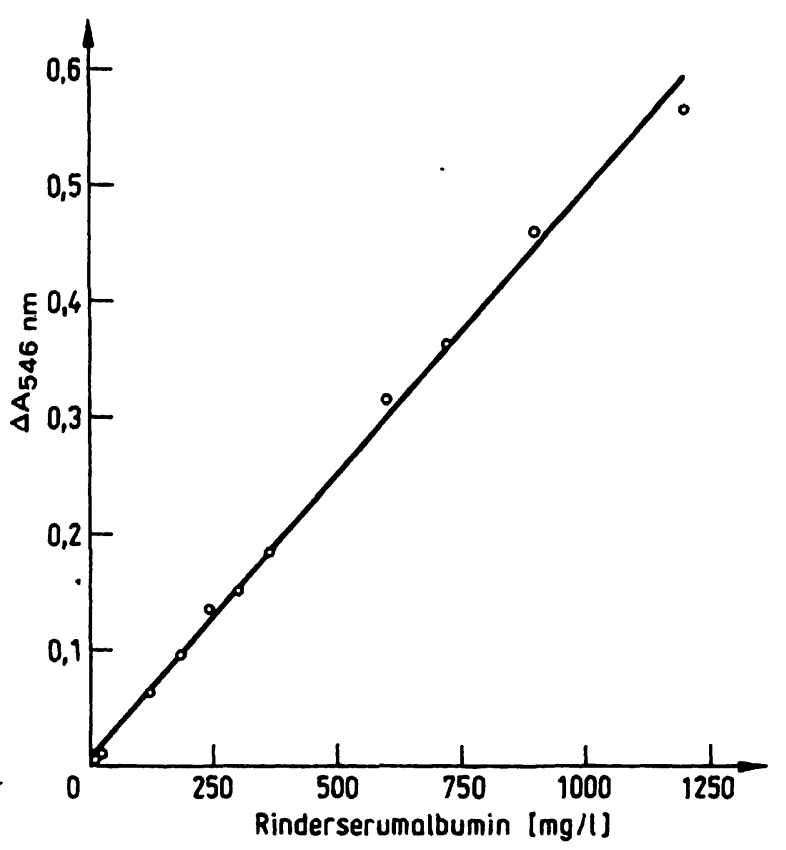

Abb. 1. Linearität der Biuret-Reaktion. Die Gleichung der Regressionsgeraden ist $\mathrm{mg} / \mathrm{l}=2129,3 \Delta \mathrm{A}+19,4$; der Korrelationskoeffizient ist $\mathrm{x}=0,999$ und die Streuung $\mathrm{s}_{\mathbf{x y}}=13,1$ $\mathrm{mg} / \mathrm{l}$.

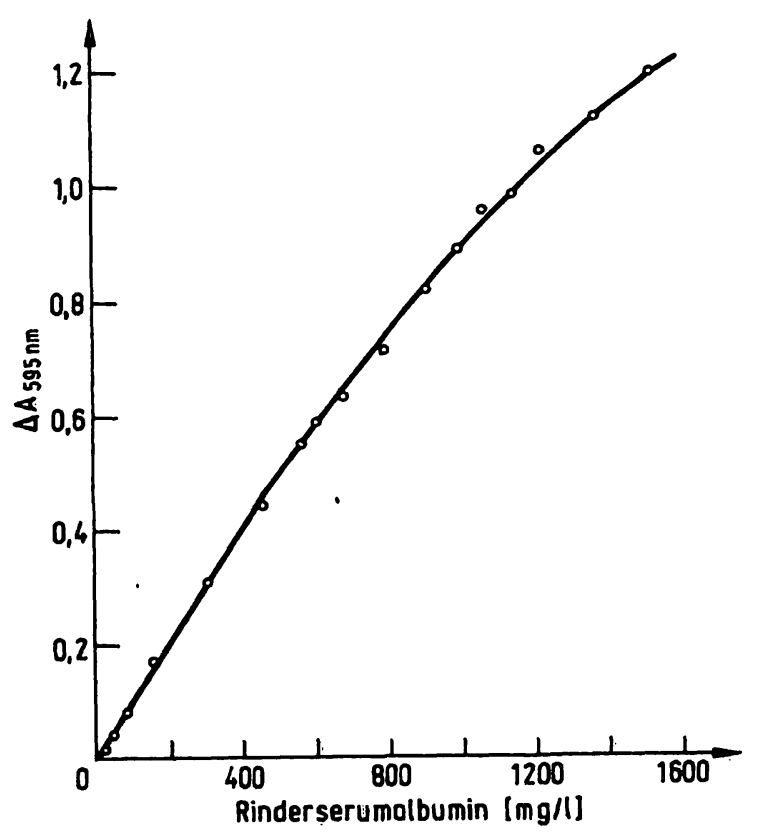

Abb. 2. Linearität der Coomassie Brilliant Blau G250-Methode. Die Gleichung der Regressionsgeraden bis zur Proteinkonzentration von $400 \mathrm{mg} / \mathrm{l}$ ist $\mathrm{mg} / \mathrm{l}=875,4 \Delta \mathrm{A}-1,3$; der Korrelationskoeffizient ist $r=0,999$ und die Streuung $s_{x y}=4,99 \mathrm{mg} / \mathrm{L}$

\section{Empfindlichkeit}

Albuminkonzentrationen von $30 \mathrm{mg} / \mathrm{l}$ in der zugegebenen Probe, entsprechend $3 \mu \mathrm{g}$ Protein im Ansatz können mit der Coomassie Brilliant Blau G250-Methode gegenüber dem Reagenzienleerwert unterschieden-werden. Die mittlere Absorptionsdifferenz beträgt 0,03 , wenn mit einer Küvette von $1 \mathrm{~cm}$ Schichtdicke gemessen wird. Die Biuret-Reaktion zeigt erst bei Rinderserumalbuminkonzentrationen ab $90 \mathrm{mg} / \mathrm{l}$, entsprechend $0,09 \mathrm{mg}$ Protein pro Ansatz einen noch gut meßbaren Absorptionsunterschied von 0,03 gegenüber dem Probenleerwert.

\section{Präzision}

Bestimmt wurde die Präzision in der Serie und die Präzision von Tag zu Tag mit 2 wäßrigen Albuminlösungen für die Coomassie Brilliant Blau G250-Methode und die Biuret-Reaktion (Tab. 1). Im gleichen Konzentrationsbereich zeigt die Coomassie Brilliant Blau G250-Methode eine eindeutig bessere Präzision. Zur Feststellung, ob die Form der Standardkurve vom verwendeten Farbreagenz abhängig ist, wurden folgende Untersuchungen durchgeführt:

1. Standardkurven unter Verwendung desselben Farbreagenzes mit Rinderserumalbumin von sowohl $2 \mathrm{Her}$ stellern als auch eines Herstellers, aber aus 2 Chargen als Doppelbestimmung erstellt.

2. Standardkurven unter Verwendung von Rinderserumalbumin der gleichen Charge, aber mit fünfmalig neu angesetztem Coomassie Brilliant Blau G250-Farbreagenz von der gleichen Herstellungscharge angefertigt.

Da die Standardkurve im Bereich von 30-400 mg/l durch eine Geradengleichung beschrieben werden kann,

Tab. 1. a) Präzision in der Serie der Coomassie Brilliant Blau G250 (CBB-G250)-Methode und der Biuret-Reaktion. Untersuchungsgut waren wäßrige Albuminlösungen in 4 verschiedenen Konzentrationen. Die Bestimmung der Präzision wurde mit 20 Werten hintereinander in jeweils einer Serie durchgeführt.

\begin{tabular}{lllll}
\hline & \multicolumn{5}{c}{ Proteinkonzentration (mg/l) } \\
& 50 & 100 & 500 & 1000 \\
\hline VK\% (CBB-G250) & 6,74 & 2,91 & 1,83 & 1,52 \\
VK\% (Biuret) & - & 9,65 & 3,78 & 3,06 \\
\hline
\end{tabular}

b) Präzision von Tag zu Tag der Coomassie Brilliant Blau G250 (CBB-G250)-Methode und der BiuretReaktion mit wäßrigen Albuminlösungen an 20 aufeinanderfolgenden Arbeitstagen in Doppelwerten. Die Variationskoeffizienten wurden aus den zweiten Werten errechnet.

\begin{tabular}{llrrr}
\hline & \multicolumn{5}{l}{ Proteinkonzentration (mg/l) } \\
& \multicolumn{1}{l}{50} & 100 & \multicolumn{1}{c}{500} & 1000 \\
\hline VK\% (CBB-G250) & 8,94 & 3,77 & 2,26 & 1,76 \\
VK\% (Biuret) & - & 11,69 & 4,23 & 3,28 \\
\hline
\end{tabular}


wurden die unter 1. und 2. genannten Standardkurven für diesen Bereich unter Verwendung von 10 Standardlösungen mit ansteigender Konzentration angefertigt. Die Untersuchung durch eine Kovarianzanalyse ergab für die unterschiedlichen Albumine eine gemeinsame Regressionsgerade von $\mathrm{mg} / \mathrm{l}=913,3 \Delta \mathrm{A}-14,3$ mit der Streuung $s_{x y}=9,8 \mathrm{mg} / \mathrm{l}$ und dem Korrelationskoeffizienten $r=0,996$. Die Werte der Kovarianzanalyse für neu angesetzte Coomassie Brilliant Blau G250-Farbreagenzlösungen sind $\mathrm{mg} / \mathrm{l}=911,2 \Delta \mathrm{A}-4,8 ; \mathrm{s}_{\mathbf{x y}}=9,2$ $\mathrm{mg} / \mathrm{l}, \mathrm{r}=0,997$. Die Werte der Korrelationskoeffizienten und der Streuung erlauben folgende Interpretation:

1. die von uns untersuchten Albumine verschiedener Hersteller und Chargen ergeben keine unterschiedlichen Standardkurven;

2. bei Verwendung von Coomassie Brilliant Blau G250Farbstoff der gleichen Charge muß nicht bei dem Ansetzen von neuem Farbreagenz eine neue Standardkurve erstellt werden. Dies wird erst notwendig, wenn die Farbstoffcharge oder der Hersteller gewechselt wird.

\section{Untersuchung an Harnen}

\section{Präzision}

Die Präzision in der Serie wurde mit 3 frisch gewonnenen Spontanharnen und 20 Werten in Serie durchgeführt (Tab. 2). Verglichen mit der Biuret-Reaktion zeigt die Coomassie Brilliant Blau G250-Methode niedrigere VKWerte. Die Bestimmung der Präzision von Tag zu Tag ist aufgrund rasch eintretender Harnzersetzung über den erforderlichen Zeitraum von 20 Tagen nicht möglich und wurde deshalb nicht durchgeführt.

\section{Spezifität}

Zur Prüfung der Spezifität der Coomassie Brilliant Blau G250-Methode wurden 10 Sammelharne mit einer Proteinkonzentration zwischen 100 und $200 \mathrm{mg} / 1$ durch Zugabe von $300 \mathrm{mg}$ Rinderserumalbumin aufgestockt und die Proteinkonzentration vorher und nachher durch Doppelbestimmung ermittelt. Die Wiederauffindung betrug $98,6 \pm 2.8 \%$.

Tab. 2. Präzision in der Serie der Coomassie Brilliant Blau G250 (CBB-G250)-Methode und der Biuret-Reaktion. Untersuchungsgut waren 3 Spontanharne mit unterschiedlichen Proteinkonzentrationen. Die Bestimmung der Präżision wurde mit 20 Werten hintereinander in jeweils einer Serie durchgeführt.

\begin{tabular}{lllll}
\hline & \multicolumn{2}{l}{$\begin{array}{l}\text { CBB-G250- } \\
\text { Methode }\end{array}$} & Biuret-Reaktion \\
& $\overline{\mathbf{x}}(\mathrm{mg} / \mathrm{l})$ & $\mathrm{VK}(\%)$ & $\overline{\mathrm{x}}(\mathrm{mg} / \mathrm{l})$ & VK (\%) \\
Ham I & 106 & 4,9 & 138 & 11,3 \\
Harn II & 284 & 3,1 & 242 & 5,6 \\
Harn III & 637 & 2,8 & 718 & 4,2 \\
\hline
\end{tabular}

\section{4h-Proteinausscheidung gesunder Probanden}

Bei einem Kollektiv von 49 klinisch gesunden Probanden mit kompletter internistischer Untersuchung und normalen Laborwerten lagen 14 unterhalb der sicher meßbaren Nachweisbarkeitsgrenze von $30 \mathrm{mg} / \mathrm{l}$, das restliche Kollektiv von 35 Probanden zeigte höhere Werte. Diese verteilen sich log-normal und lassen sich durch folgende Kennziffern beschreiben:

Median $=61,0 \mathrm{mg} / 24 \mathrm{~h}$, Streufaktor $=1,966$, zentrale $90 \%$-Masse $=31,2-120,0 \mathrm{mg} / 24 \mathrm{~h}$, Mittelwert $\mu=76,6$ $\mathrm{mg} / 24 \mathrm{~h}$, Dichtemittel $=38,7 \mathrm{mg} / 24 \mathrm{~h}, 95 \%$ Vertrauensbereich für $\mu=48,5-77,0 \mathrm{mg} / 24 \mathrm{~h}$. Aufgrund der vorliegenden logarithmischen Verteilung wird der Normalbereich nicht mit den üblichen $2 \mathrm{~s}$-Schranken angegeben, sondern mit der zentralen 90\%-Masse definiert. Danach ergibt sich ein Normalbereich von 31,2-120,0 mg/24 h. Die Werte sind gültig, wenn zur Kalibrierung Rinderserumalbumin eingesetzt wird.

Aufgrund der Unempfindlichkeit der Biuret-Reaktion war die Ermittlung eines Normalbereiches mit diesem Verfahren nicht möglich. Nur 18 Probanden zeigte eine meßbare Proteinausscheidung.

\section{Vergleichende Untersuchungen mit Patientenharnen}

Zur Beurteilung der Wertigkeit der Coomassie Brilliant Blau G250-Methode für die Protēinbestimmung in Patientenharnen wurde die Proteinkonzentration vergleichend zur Biuret-Reaktion bestimmt. Untersucht wurde der morgendliche Spontanharn von 312 Patienten mit Organleiden, Stof fwechsel- und Systemerkrankungen, die mit der Coomassie Brilliant Blau G250-Methode eine meßbare Proteinkonzentration zeigten. Nur 66,9\% der Harne hatten auch mit der Biuret-Reaktion eine meßbare Proteinkonzentration. Zum statistischen Vergleich beider Proteinbestimmungsmethoden wurden nur Hamproteinwerte eingesetzt, die mit der BiuretReaktion über $200 \mathrm{mg} / 1$ lagen, da dann eine annähernd vergleichbare Präzision mit der Coomassie Brilliant Blau G250-Methode vorliegt.

Der Vergleich beider Methoden ist anhand der standardisierten Hauptkomponenten-Methode in Abbildung 3 dargestellt (17). Die Anwwendung dieses statistischen Zusammenhangsmodells ist vorteilhaft, wenn zwei Methoden miteinander verglichen werden, deren Meßwerte $x$ und $y$ als mit zufälligen Fehlern behaftet angesehen werden (18). Zwischen beiden Methoden besteht eine gute Korrelation mit einem Korrelationskoeffizienten $r=0,966$. Die Gleichung der orthogonalen Regressionsgeraden $X_{0} ; y=0,83 x+8,7 \mathrm{mg} / 1$ gibt an, daß $0,83 \mathrm{mg}$ Hamprotein, gemessen mit der Coomassie Brilliant Blau G250-Methode, 1,0 mg Protein, bestimmt mit der BiuretReaktion, entsprechen. Ersichtlich wird auch, daß für beide Methoden die obere Normalbereichsgrenze der Proteinausscheidung nicht identisch sein kann. 


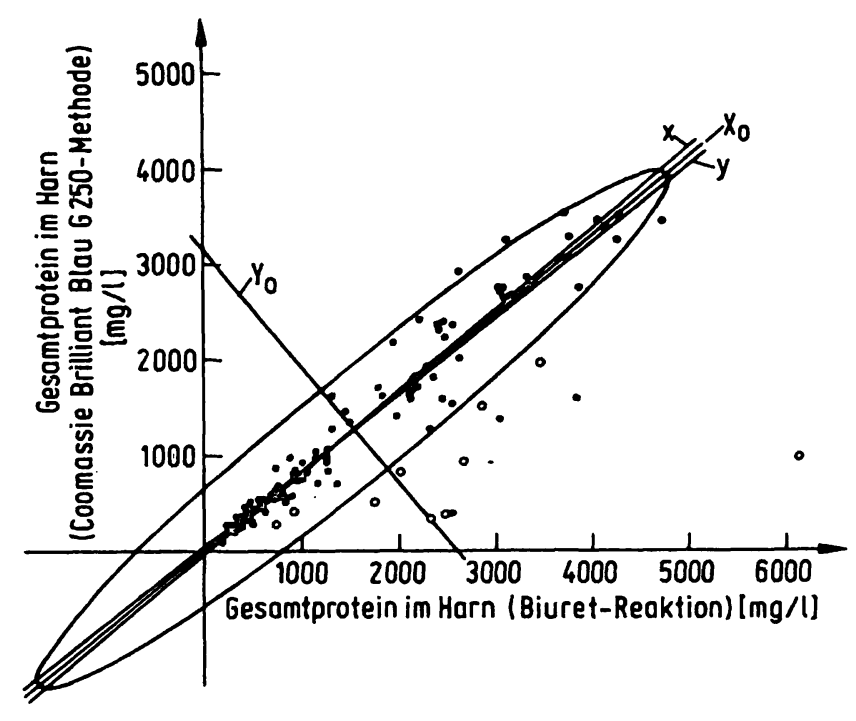

Abb. 3. Methodenvergleich für Gesamtprotein im Harn nach der stand ardisierten Hauptkomponentenmethode für $n=134$ Patienten mit einer Proteinurie über $200 \mathrm{mg} / \mathrm{l}$ Die orthogonalen Regressionsgeraden haben folgende Gleichungen: $\mathrm{X}_{\mathrm{o}} ; \mathrm{y}=0,827 \mathrm{x}+8,7 \mathrm{mg} / 1\left(\mathrm{X}_{\mathrm{O}}=\right.$ standardisierte Hauptkomponente), $Y_{0} ; y=-1,209 x+3125,3 \mathrm{mg} / 1$. Der Korrelationskoeffizient ist $r=0,966$, die Streuung um die standardisierte Hauptkomponente $s_{X y}=277,0 \mathrm{mg} / \mathrm{L}$ Die Gleichungen der Regressionsgeraden sind $y=0,804 x$ $+43,7 \mathrm{mg} / 1$ und $\mathrm{x}=1,161 \mathrm{y}+51,0 \mathrm{mg} / \mathrm{l}$. Die Konzentrationsellipse umfaßt den $95 \%$ Toleranzbereich und die Längen der beiden Halbachsen sind $a=6188,5 \mathrm{mg} / \mathrm{l}$ und $\mathrm{b}=539,6 \mathrm{mg} / \mathrm{l}$. Die Seitenlängen des umschreibenden Rechtecks sind: horizontale Seite $=6429,5 \mathrm{mg} / \mathrm{l}$ und vertikale Seite $5402,6 \mathrm{mg} / \mathrm{l}$.

Die mit einem Kreis gekennzeichneten 11 Harnwerte wurden nicht in die Berechnung mit einbezogen. Ës handelt sich um Myelompatienten mit immunelektrophoretisch nachgewiesener Bence-Jones-Proteinurie, die in der Urineiweiß-Elektrophorese auf Celluloseacetatfolie nahezu ausschließlich einen M-Gradienten bei keiner oder nur geringer Albumin- oder Globulinausscheidung zeigten.

Bei der klinischen Auswertung des Kollektivs wurden in den der Biuret-Achse zugekehrten Randpartien der Konżentrationsellipse und außerhalb darunter liegend gehäuft Proteinurien bei Diabetikern gefunden. In den der Coomassie Brilliant Blau G250-Achse zugekehìten Randpartien der Konzentrationsellipse und im außerhalb darüber liegenden Feld findet man gehäuft Proteinurien bei hochgradiger Niereninsuffizienz.

\section{Einfluß des Proteinurie-Typs}

Werden Harnproteine mit der SDS-PolyacrylamidgelElektrophorese bei pH-Wert 7,2 aufgetrennt (16), so zeigen tubuläre Proteinurien ein Bandenmuster von Proteinen, das anodisch der Albuminfraktion liegt und deshalb molekulargewichtsbezogen kleiner als Albumin ist. Bei der glomerulären Proteinurie liegt das Muster kathodisch der Albuminfraktion, die gemischte
Proteinurie zeigt eine Kombination beider Muster (19). Von 23 Patienten mit gemischter, glomerulärer und mit tubulärer Proteinurie (außer Bence-Jones-Protein) wurde das Gesamtprotein mit der Biuret-Reaktion und der Coomassie Brilliant Blau G250-Methode ermittelt und die Werte in Abbildung 3 mit aufgenommen. Bis auf einen der Werte liegen alle in der Konzentrationsellipse, was darauf hinweist, daß bei den klinisch relevanten Proteinurien der Typ auf die Gesamtproteinbestimmung mit der Coomassie Brilliant Blau G250Methode keinen Einfluß hat.

\section{Diskussion}

Der Begriff Harnprotein ist keine wohl definierte Einheit, sondern umfaßt eine große Gruppe von Proteinen, deren Zusammensetzung wechselt. Die in der Literatur angegebenen unterschiedlichen Normalbereichsgrenzen der täglichen Proteinausscheidung sind vorwiegend auf die Proteinbestimmungsmethoden zurückzuführen. So wird z. B. für die turbidimetrischen Techniken ein Bereich von $25-75 \mathrm{mg} / 24 \mathrm{~h}$ (10), für die BiuretReaktion von $40-150 \mathrm{mg} / 24 \mathrm{~h}$ (20) und für die FolinLowry-Methode von $38-394 \mathrm{mg} / 24 \mathrm{~h}$ (21) angegeben. Ursache ist das nicht einheitliche chemische Verhalten der einzelnen Proteine in der Bestimmungsreaktion, die unspezifische Mitreaktion von Nichtproteinen sowie eine ungenügende Präzipitation der Urineiweiße. So ergibt bei den turbidimetrischen Techniken Albumin eine andere Trübung mit Sulfosalicylsäure als Globuline (10). Falsch hohe Werte täuscht die Biuret-Reaktion vor bei Anwesenheit von Pigmenten sowie Stickstoff-, Sauerstoff- und Schwefel-haltigen Substanzen, die mit $\mathrm{Cu}-$ Ionen einen Komplex bilden. Noch stärker gestört wird die Folin-Lowry-Methode, neben Pigmenten gehen alle Substanzen in die Reaktion ein, die eine Reduktion des Folin-Lowry-Reagenzes bewirken. 4-20\% der Harnproteine sind mit Trichloressigsäure nicht präzipitierbar, der Wirkungsgrad der Fällung nimmt mit sinkender Proteinkonzentration ab (22). Somit werden falsch niedrige Werte bei allen Bestimmungsreaktionen vorgetäuscht, denen eine Proteinfällung vorangeht.

Der Coomassie Brilliant Blau G250-Methode geht keine Proteinfällung voraus. Nach Zugabe der Probe zum Farbreagenz reagiert das Farbstoffanion mit den $\mathrm{NH}_{3}^{+}$Gruppen der Proteine unter Bildung eines blauen Farbstoff-Protein-Komplexes. Da jedoch die unterschiedlichen Proteine des Harnes nicht die gleiche Anzahl von $\mathrm{NH}_{3}^{+}-$ Gruppen tragen und auch nicht einheitlich mit den Farbstoffanionen reagieren, zeigen sie eine verschiedene Farbstoffbindung und damit keinen gemeinsamen $\mathrm{Ab}$ sorptionskoeffizienten. Zur Messung von Proteinlösungen wechselnder Zusammensetzung wie Harn ist die Coomassie Brilliant Blau G250-Methode deshalb theoretisch nicht geeignet, es sei denn, eine Lösung identischer 
Proteinverteilung dient als Referenz. Bei den klinisch relevanten Proteinurien liegt, ausgenommen der selektiven Bence-Jones-Protein-Ausscheidung, nicht die excessive Vermehrung eines Einzelproteins, sondern die Ausscheidung eines Proteinmusters vor. Aus diesem Grunde ist die unterschiedliche Proteinbindung von Coomassie Brilliant Blau G250 nicht von wesentlicher Bedeutung für die Gesamtproteinbestimmung im Harn, wie der Vergleich der Coomassie Brilliant Blau G250Methode mit der Biuret-Reaktion in Abbildung 3 zeigt.

Die gegenüber der Biuret-Reaktion im Mittel 4 fach höhere Empfindlichkeit der Coomassie Brilliant Blau G250-Methode ist besonders wertvoll bei fraglichen Proteinurien, z. B. Sulfosalicylsäureprobe positiv, Teststreifenverfahren negativ oder schwach positiv. Weitere Schwerpunkte sind die Verlaufsbeurteilung der Proteinausscheidung bei Schwangerschaft sowie die Erkennung toxischer Nierenschädigung unter Therapie mit Arzneimitteln.

Die von uns an einem kleinen Kollektiv ermittelte obere Normbereichsgrenze der $24 \mathrm{~h}$-Proteinausscheidung liegt etwa um $30 \mathrm{mg}$ unter den für die Biuret-Reaktion in der Literatur angegebenen Werten (3). Dies mag zum einen darin begründet sein, daß unter Bezugnahme auf Rinderserumalbumin als Referenzprotein $1 \mathrm{mg} \mathrm{Ham}$ protein, gemessen mit der Biuret-Reaktion, nur 0,8 mg in der Coomassie Brilliant Blau G250-Methode entsprechen. Zum anderen werden mit der Coomassie Brilliant Blau G250-Methode nur Peptide mit einem Molekulargewicht $M_{\mathrm{r}}>3000$ gemessen (23) und nicht die niedermolekularen Urinpeptide wie mit der BiuretReaktion.

\section{Literatur}

1. Foster, P. W., Rick, J. J. \& Wolfson, W. Q. (1952), J. Lab. Clin. Med. 39, 618-623.

2. Piscator, M. (1962), Arch. Environ. Health 5, 325-331.

3. Savory, J., Pu, H. P. \& Sunderman Jr., F. W. (1968), Clin. Chem. 14, 1160-1171.

4. Hiller, A., Greif, R. L. \& Beckman, W. W. (1948), J. Biol. Chem. 176, 1421-1429.

5. Poortsmans, J. \& van Kerchove, E. (1963), Clin. Chim. Acta $8,485-488$.

6. Henry, R. J., Sobel, C. \& Segalove, M. (1956), Proc. Soc. Exp. Biol. Med. 92, 748-751.

7. Lowry, O. H., Rosebrough, N. J., Farr, A. L. \& Randall, R. J. (1951), J. Biol. Chem. 193, 265-275.

8. Saifer, A. \& Gerstenfeld, S. (1964), Clin. Chem. 10, 321-334.

9. Doetsch, K. \& Gadsden, R. H. (1973), Clin. Chem. 19; 1170-1178.

10. Henry, R. J., Cannon, D. C. \& Winkelman, J. W. (1974), Clinical Chemistry, Principles and Technics, S. 431, Harper and Row, New York.

11. Bradford, M. M. (1976), Anal. Biochem. 72, 248-254.

12. Schleicher, E. \& Wieland, O. H. (1978), J. Clin. Chem. Clin. Biochem. 16, 553-554.
Die bei Bence-Jones-Proteinurien ermitteltèn falsch niedrigen Werte der Coomassie Brilliant Blau G250Methode stellen das Verfahren nicht in Frage, da die Empfindlichkeit von etwa $60 \mathrm{mg} / 1 \mathrm{immer}$ noch günstiger ist als bei der Biuret-Reaktion. Die Ursache der mangelnden Ǔbereinstimmung zwischen beiden Proteinbestimmungsmethoden bei einem Teil der Diabetiker und hochgradig Niereninsuffizienten bleibt ungeklärt.

Harneigene Nichtproteinsubstanzen stören die Coomassie Brilliant Blau G250-Methode nicht, wenn Probenvolumen von $0,10 \mathrm{ml}$ und niedriger eingesetzt werden. Bei Analyse eines Harnes mit Einsatz unterschiedlicher Probenvolumina werden volumenkorrigiert identische Proteinkonzentrationen ermittelt.. Störende harnfremde Nichtproteinsubstanzen sind Detergentien, Toluol und Antibiotika in hoher Konzentration (24). Harne mit einem $\mathrm{pH}$-Wert $<4$ täuschen erniedrigte, solche mit einem pH-Wert $>8$ erhöhte Werte vor $(14,24)$.

Unsere Ergebnisse zeigen, daß die Coomassie Brilliant Blau G250-Methode der Biuret-Reaktion vergleichbare Hamproteinwerte liefert. Sie zeichnet sich jedoch gegenüber dieser Methode durch eine einfachere Testdurchführung, bessere Präzision und höhere Empfindlichkeit aus.

\section{Danksagung}

Die Autoren danken Herrn Diplommathematiker Dr. H. L. Christl für die Unterstützung bei den statistischen Ausarbeitungen.

13. Nesbitt, J., Sauer, D. \& Culpepper (1978), J. Amer. Med. Technol. 40, 278-279.

14. McIntosh, J. C. (1977), Clin. Chem. 23, 1939-1940.

15. Thomas, L. (1981), Eiweißelektrophorese, Verlag Urban \& Sch warzenberg, S. 99-118.

16. Virella, G. \& Pires, M. T. (1973), in: Protides of the Biological Fluids (Peeters, H., ed.), S. 407-417.

17. Wissenschaftl Tabellen (1973), Ciba Geigy S. 173-184.

18. Haeckel, R. \& Schneider, B. (1980), GIT 3, 99-104.

19. Walb, D. (1979), Med. Welt 30, 210-215.

20. Boyce, W. H., Garvey, F. K. \& Norfleet, C. M. (1954), J. Clin. Invest. 33, 1287-1297.

21. Hemmingsen, L. \& Skov, F. (1968), Clin. Chim. Acta 19, 81-87.

22. Beckman, W. W., Hiller, A., Schedlowsky, T. \& Archibald, R. M. (1943), J. Biol Chem. 148, 247-248.

23. Sedmark, J. J. \& Grossberg, S. E. (1977), Anal. Biochem. $79,544-552$.

24. Pierce, J. \& Suelter, C. H. (1977), Anal. Biochem. 81, 478-480. 